\title{
Effect of short-term cryostimulation on antioxidative status and its clinical applications in humans
}

\author{
Elżbieta Miller • Lukasz Markiewicz • \\ Joanna Saluk $\cdot$ Ireneusz Majsterek
}

Received: 23 May 2011 / Accepted: 8 August 2011 / Published online: 28 August 2011

(C) The Author(s) 2011. This article is published with open access at Springerlink.com

\begin{abstract}
Whole body cryostimulation (WBCT) is becoming popular in medicine and sport as an adjuvant form of treatment since late 1970s. Only a few works concerning antioxidant protection after WBCT have been published. The aim of this study was to determine the effect of a ten 3-min-long exposures (one exposure per day) to cryogenic temperature $\left(-130^{\circ} \mathrm{C}\right)$ on the level of total antioxidant status (TAS), activity of selected antioxidant enzyme superoxide dismutase (SOD) and main non-enzymatic antioxidant-uric acid (UA) in WBCT study group (man $n=24$; female $n=22$ ) and non-WBCT control subjects (man $n=22$; female $n=26$ ). Moreover, we evaluated the lipid peroxidation measured as thiobarbituric acid reactive substances products. Their blood samples were collected twice at an interval of 10 days in both study group and control subjects. The activity of antioxidant enzyme and lipid
\end{abstract}

Communicated by William J. Kraemer.

\section{E. Miller}

Rehabilitation Ward, III General Hospital in Lodz,

Milionowa 14, Lodz, Poland

E. Miller $(\square)$

Łyżwiarska 31, 94-124 Lodz, Poland

e-mail: betty.miller@interia.pl

J. Saluk

Department of General Biochemistry,

University of Lodz, Banacha 12/16, Lodz, Poland

e-mail: juszczak@biol.uni.lodz.pl

\section{Ł. Markiewicz · I. Majsterek}

Department of Chemistry and Clinical Biochemistry,

Medical University of Lodz, Pl. Hallera 1, Lodz, Poland

e-mail: lukasz.markiewicz@umed.lodz.pl

I. Majsterek

e-mail: ireneusz.majsterek@umed.lodz.pl peroxidation was assayed in erythrocytes, while the concentration of uric acid was measured in plasma. After completing a total of ten WBCT sessions a significant increase $(p<0.001)$ of TAS and UA levels in plasma $(p<0.001)$ in comparison to non-WBCT was observed. Our data showed that there was statistically significant increase of the activities of SOD in erythrocytes obtained from WBCT study group compared to non-WBCT controls after 10 days of treatment $(p<0.001)$. It was concluded that expositions to extremely low temperatures use in cryostimulation improve the antioxidant capacity of organism.

Keywords Whole body cryostimulation - Antioxidant therapy $\cdot$ Total antioxidant status $\cdot$ Lipid peroxidation

\section{Introduction}

Whole body cryostimulation (WBCT) is becoming popular in medicine and sport as an adjuvant form of treatment. The reported reasons for WBCT include improved general wellbeing, and self-treatment or body hardening against respiratory tract infections and musculoskeletal pains. WBCT is often used as an additional form of treatment for inflammation and pain in, for example, arthritis, osteoarthritis, and fibromyalgia (Duqué et al. 2005; Miller et al. 2010a, b, c). WBCT has been found useful in neurological diseases including multiple sclerosis (Miller et al. 2010a, b, c) and psychiatric disorders such as anxiety-depressive syndrome (Rymaszewska et al. 2008). Treatment with the total exposure of the body at extremely low temperatures was first introduced in Japan towards the end of the 1970s by Yamauchi (1989) who constructed the first cryogenic chamber and successfully used cryotherapy to treat rheumatism. The influence of extremely low temperatures on the human 
body and physiological reactions is not fully recognized (Lange et al. 2008; Lubkowska et al. 2010; Miller et al. 2010a, b, c).

An imbalance between free radical generation and antioxidant defense leads to oxidative stress which can damage lipids, proteins, and nucleic acids in cells causing cell death in various cell types. Oxidative stress is a hallmark of many chronic diseases such as neurodegenerative and cardiovascular disorders. The direct estimation of reactive oxygen species (ROS) generation in vivo is difficult. A common approach to measure oxidative stress in vivo is to determinate lipid peroxidation by means of commonly used assay with thiobarbituric acid (expressed as thiobarbituric acid reactive substances, TBARS). Lipid peroxidation is one of the important phenomenon and has been implicated in a number of deleterious effects, such as increased osmotic fragility, decreased membrane rigidity, and cellular deformation (Miller et al. 2011).

The antioxidant system in the organism includes a variety of antioxidant enzymes such as superoxide dismutase (SOD), catalase (CAT), glutathione peroxidase (GPx) and non-enzymatic such as antioxidants glutathione, vitamins A, C, D, coenzyme Q, uric acid, etc. In humans, over half the antioxidant capacity of blood plasma comes from uric acid. Uric acid like ascorbic acid is a strong reducing agent and potent antioxidant responsible for total antioxidant status (TAS) level in plasma (Miller et al. 2011).

Currently, a variety of antioxidative supplementary therapy for the treatment of chronic diseases are used worldwide. There is a need for developing new therapies such as WBCT which can be used as adjuvant antioxidative therapy. The mechanisms of action of hypothermic protection are not entirely understood. It seems clear that lower temperature protects tissues deprived of oxygen by slowing the rate of cellular damage that occurs from the formation of free radicals, chemical metabolites, and tissue edema. According to increasing evidence, hypothermia can significantly improve outcomes of diseases with oxidative stress background such as neonates hypoxic-ischemic encephalopathy (Perrone et al. 2010), brain injury (Varon et al. 2011), multiple sclerosis (Miller et al. 2010a, b, c), depression (Miller et al. 2010a, b, c) and cerebral ischemia (Varon et al. 2011). So, it seems important to know the influence of repeated exposure to the stress induced by cryogenic temperatures affecting the whole body of healthy people in order to use this knowledge efficiently in clinical practice.

Only a few works concerning antioxidant protection after ten sessions of WBCT $\left(-130^{\circ} \mathrm{C}\right)$ in healthy men have been published (Duqué et al. 2005; Lubkowska et al. 2009). Recent reports suggest a stimulating antioxidative effect of cryostimulation but there is no information regarding the substances responsible for it (Miller et al. 2010a, b, c).
The antioxidants such as uric acid and the antioxidative enzymes such as superoxide dismutase (CuZnSOD) protect organisms (Lubkowska et al. 2009; Miller et al. 2010a, b, c) against production of free radicals and different reactive oxygen and nitrogen species (ROS/RNS). The measurement of TAS in plasma represents the body redox status better than the measurement of the single circulating antioxidant.

We hypothesized that the generation of ROS/RNS may be partly inhibited by ten sessions of WBCT (Miller et al. $2010 a, b, c)$. Therefore, the aim of this study was to determine the effect of ten sessions of 3-min-long exposures (one exposure per day) to cryogenic temperature $\left(-130^{\circ} \mathrm{C}\right)$ on TAS, the activity of selected antioxidant enzyme superoxide dismutase (CuZnSOD) and main non-enzymatic antioxidant-uric acid (UA) in the middle age healthy subjects. Moreover, we evaluated the lipid peroxidation measured as TBARS.

\section{Materials and methods}

\section{Subjects presentation}

Forty-six healthy subjects ( $\operatorname{man} n=24$; female $n=22$ ) (mean age $37.5 \pm 3.1$ years; body mass index (BMI) $26.9 \pm 4.2$; height $171 \pm 2.1 \mathrm{~cm}$; weight $76.1 \pm 9.7 \mathrm{~kg}$; body surface area (BSA) $1.77 \pm 4.2 \mathrm{~m}^{2}$ (female), $1.98 \pm 3.8 \mathrm{~m}^{2}$ (male); Table 1) were chosen for the study group (WBCT). The subjects were matched pairwise, having similar BMI, age, physical activity and use of hormonal contraception (6 females as well as in the study and control group). None of the subjects had practiced WBCT regularly before the study. The subjects were moderately physically active, and no outdoor workers were included. They were asked to maintain their physical activity habits during the study period. Non-WBCT group (man $n=22$; female $n=26$ ) (mean age $37.9 \pm 2.1$ years; body mass index (BMI) $27.1 \pm 4.1 ;$ height $169.9 \pm 9.2 \mathrm{~cm}$; weight $78.1 \pm 9.1 \mathrm{~kg}$; body surface area (BSA) $1.76 \pm 3.2 \mathrm{~m}^{2}$ (female), $1.97 \pm 4.5 \mathrm{~m}^{2}$ (male); $p>0.05$ ) was also included in the study as the control group to measure blood markers before and 10 days later without any intervention to eliminate the possibility of changes between days.

Their blood samples were collected twice at an interval of 10 days similar to the study group but without WBCT treatment. All examined subjects as well as controls received no immunomodulators, immunostimulators, hormones, vitamins, minerals or any other substitutions with antioxidative properties. Prior to the study, all the subjects had undergone medical checkups including a resting ECG.

Each participant gave his written consent before participation in the research, and the Bioethical Committee of the 
Table 1 Characteristics of study subjects

\begin{tabular}{lcc}
\hline & Females $(n=22)$ & Males $(n=24)$ \\
\hline Mean age (years) & $38 \pm 3$ & $37 \pm 5$ \\
BMI $\left(\mathrm{kg} / \mathrm{m}^{2}\right)$ & $24 \pm 4$ & $27.7 \pm 4$ \\
Height $(\mathrm{cm})$ & $165 \pm 8$ & $172 \pm 8$ \\
Weight $(\mathrm{kg})$ & $68 \pm 10$ & $82 \pm 12$ \\
BSA $\left(\mathrm{m}^{2}\right)$ & $1.77 \pm 4.2$ & $1.98 \pm 3.8$ \\
\hline
\end{tabular}

$B M I$ body mass index, $B S A$ body surface area

Medical University of Lodz, Poland issued their formal consent, according to the Declaration of Helsinki. The study was performed in the Department of Biochemistry, University of Lodz, Poland and Rehabilitation Division, III General Hospital in Lodz, Poland.

\section{Experimental design}

An experimental trial with WBCT consisted of the 46 (man $n=24$; female $n=22$ ) subjects exposed by ten sessions of WBCT (one session per day) in a cryogenic chamber carried out daily from Monday to Friday. The used cryogenic chamber (KR2005N, liquid nitrogen as a coolant) had two rooms: the vestibule, with the temperature of $-60^{\circ} \mathrm{C}$, and the main chamber, with temperature $-130^{\circ} \mathrm{C}$. Sessions in the chamber lasted $3 \mathrm{~min}$ according to the guidance of Gregorowicz and Zagrobelny (2007) on the appropriated duration of exposure and temperature for adult patients and a list of medical conditions in which WBCT is unsuitable. During WBCT, the whole subjects group wore the same bathing suits, surgical masks, caps, gloves, socks, and shoes. WBCT sessions were applied everyday between 10.30 and $11.30 \mathrm{a} . \mathrm{m}$. The participants were exposed to sessions of extremely low temperature $\left(-130^{\circ} \mathrm{C}\right)$ in a cryogenic chamber. Subjects entered the main chamber in groups of five or four. In the cryogenic chamber, the subjects were instructed to move slowly, walking in a circle, one behind another, without verbal contact. Just before each session of cryostimulation, systolic and diastolic blood pressure was measured. In all 46 subjects completed the WBCT treatment. No illnesses or side effects occurred during the study period.

The study was carried out from March to November 2010. Observations were examined at two stages: at the beginning and at the end of the study.

In all the groups, blood samples $(2.4 \mathrm{ml})$ were obtained from an antecubital forearm vein using vacutainer system tubes. Blood samples were collected into cooled EDTA and centrifuged to isolate plasma and erythrocytes. Samples of blood were taken just before the first 10 days cycle of therapy and directly after the last immersion.
Biochemical investigations

\section{Measurement of uric acid}

Blood samples were analyzed at the SYNEVO Laboratory at III General Hospital in Lodz. The concentration of uric acid was determined using a colorimetric enzyme assay on the Cobas Integra 400 plus analyzer. Results are expressed as $\mathrm{mg} / \mathrm{dl}$ of uric acid. References range is $2.5-8.0 \mathrm{mg} / \mathrm{dl}$.

\section{Measurement of CuZnSOD activity}

Superoxide dismutase activity in erythrocytes was measured according to Misra and Fridovich method (1972). The absorbance of the examined samples was estimated at $380 \mathrm{~nm}$ (using Beckman spectrophotometer) at $37^{\circ} \mathrm{C}$. The activity was expressed as $\mathrm{U} / \mathrm{gHb}$. Superoxide dismutase (CuZnSOD) is within the reference range. The body utilizes this enzyme to rapidly convert the superoxide anion radical to hydrogen peroxide, which is less toxic to cells. References range is 1334.34-2351.11 (U/g Hb) (Mrowicka et al. 2008).

\section{Measurement of TAS}

Determination of TAS in blood plasma was performed by spectrophotometric method according to procedure no. NX2332 by Randox (Randox Laboratories Ltd. Ardmore, Diamond Road, Crumlin, Co Antrim, UK, BT29 4QY). Briefly, ABTS (2,2'-Azino-di-[3ethylbenzthiazoline sulphonate]) was incubated with peroxide (metmyoglobin) and $\mathrm{H}_{2} \mathrm{O}_{2}$ to produce the radical cation ABTS with a relatively stable blue-green colour. Antioxidants when added in examined sample caused suppression of this color production measured as decrease of absorbance with a spectrometer (UV/VIS Spectrometer Lambda 14P, Perkin Elmer, USA) at $600 \mathrm{~nm}$. TAS was calculated as concentration of antioxidants (mmol/l). References range is $8.30-1.77 \mathrm{mmol} / \mathrm{l}$ plasma. This range was measured in a European working population. It is recommended that each laboratory establish its own reference range to reflect the age, sex, diet, and geographical location of the population.

\section{Measurement of TBARS}

For TBARS estimation in plasm, samples of plasma were transferred to an equal volume of $20 \%(\mathrm{v} / \mathrm{v})$ cold trichloroacetic acid in $0.6 \mathrm{M} \mathrm{HCl}$ and centrifuged at $1200 \times g$ for 15 min. One volume of clear supernatant was mixed with 0.2 vol. of $0.12 \mathrm{M}$ thiobarbituric acid in $0.26 \mathrm{M}$ Tris, $\mathrm{pH} 7.0$ and immersed in a boiling water bath for $15 \mathrm{~min}$. Absorbance at $532 \mathrm{~nm}$ was measured and the results are 

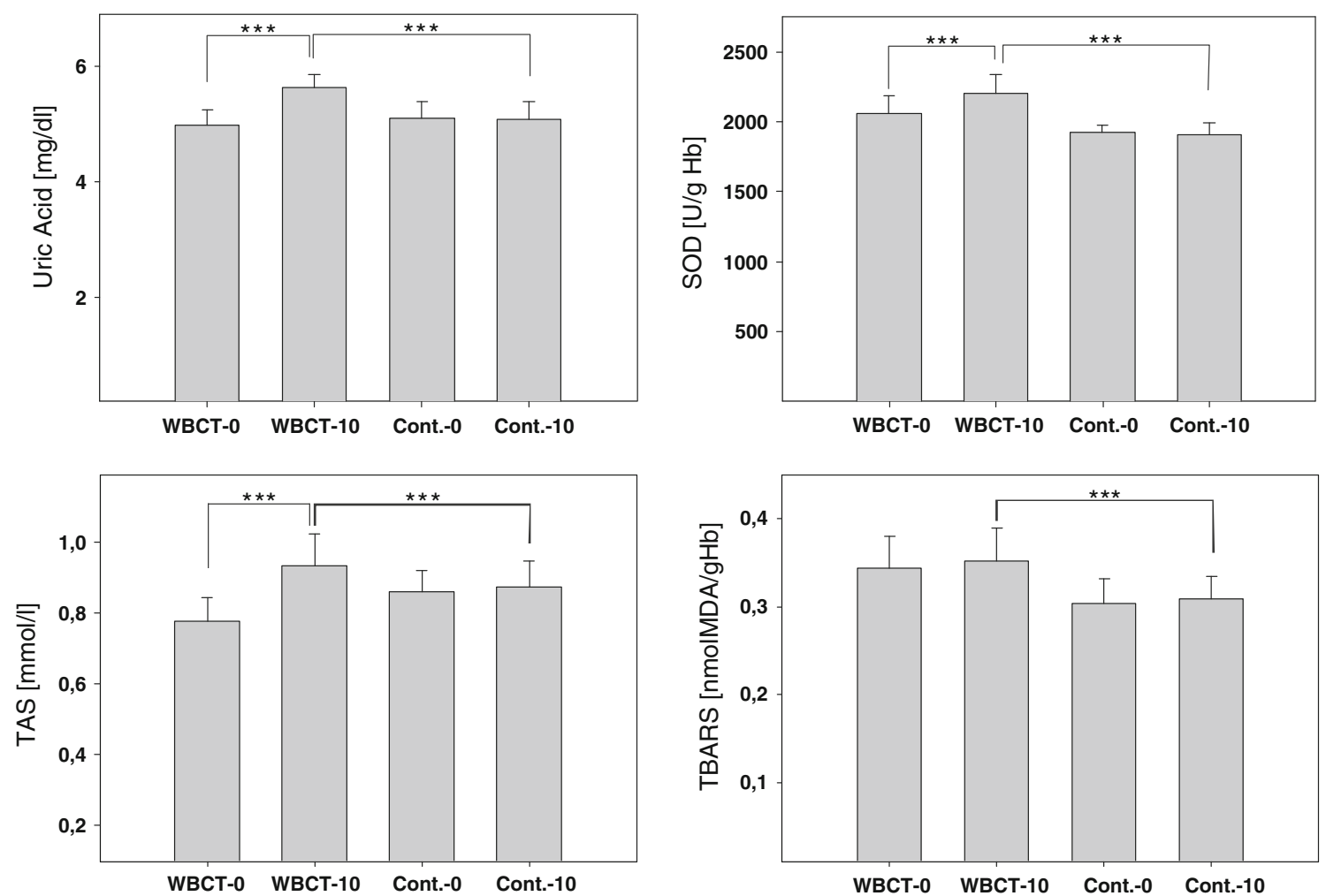

Fig. 1 The effect of whole body cryostimulation (WBCT) on the level of uric acid (UA), total antioxidant status (TAS), thiobarbituric acid reactive substances (TBARS) in plasma and superoxide dismutase (SOD) activity in erythrocytes of WBCT study group before (WBCT-0)

expressed as $\mu \mathrm{mol}$ of TBARS. Normal human plasma has lipid peroxide level of range 1.86-3.94 $\mu \mathrm{mol}$.

\section{Statistical analysis}

The results were statistically elaborated. An enzyme activity as well as plasma uric acid level was expressed as mean value $\pm \mathrm{SD}$. $10 \%$ of the samples were blinded and replicated for quality control (QC). If no significant differences between variations were found, as assessed by the Snedecor-Fisher test, the differences between the two groups were evaluated by applying the Student's $t$-test. Otherwise, the Cochran-Cox test was used. The data were analyzed using the STATISTICA (StatSoft, Tulsa, OK) statistical package. Differences were considered significant, when the significance was $p<0.05$.

\section{Results}

Firstly, our study showed a statistically significant increase of $10 \%$ of the level of UA in plasma obtained from WBCT study group compared to non-WBCT controls after 10 days of treatment $(p<0.001)$ (Fig. 1; Table 3). We also showed

and after 10 days of cryostimulation (WBCT-10) as well as nonWBCT control subjects before (Cont.-0) and 10 days after (Cont.-10); Error bars denote standard deviation (SD). $\left({ }^{*} p<0.05, * * p<0.01\right.$, $* * * p<0.001)$

that there was statistically significant increase of $13 \%$ of the UA level in WBCT study group after 10 days of treatment compared to the initially taken sample in this group $(p<0.001)$ (Fig. 1; Table 3). Moreover, after WBCT the increase of UA was about $14 \%$ in women and $10 \%$ in men $(p<0.0001)$ (Table 2). There were no statistically significant increase of UA levels in the non-WBCT control group after 10 days when compared to the initially taken sample in this group $(p>0.05)$ (Fig. 1; Table 3).

Secondly, data obtained showed that there was statistically significant increase of $15 \%$ of the activities of CuZnSOD in erythrocytes obtained from WBCT study group compared to non-WBCT controls after 10 days of treatment $(p<0.001)$ (Fig. 1; Table 3). There was also statistically significant increase of $6 \%$ of the activities of CuZnSOD in erythrocytes obtained from WBCT study group after 10 days of treatment, compared to the initially taken sample in this group $(p<0.001)$ (Fig. 1; Table 3). The activities of CuZnSOD in erythrocytes after cryostimulation were also increased by about $6 \%$ in both sexes $(p<0.0001)$ (Table 2). Moreover, there were no statistically significant increase of the activities of CuZnSOD in erythrocytes obtained from non-WBCT control group after 10 days when compared to the initially taken sample in this group $(p>0.05)$ (Fig. 1; Table 3). 
Table 2 Effect of whole body cryostimulation (WBCT) on TBARS (thiobarbituric acid reactive substances), superoxide dismutase (SOD), total antioxidant status (TAS), and uric acid in men and women

\begin{tabular}{|c|c|c|c|c|c|c|}
\hline & \multicolumn{2}{|l|}{ Females $(n=22)$} & \multirow[t]{2}{*}{$p$} & \multicolumn{2}{|l|}{ Males $(n=24)$} & \multirow[t]{2}{*}{$p$} \\
\hline & Before WBCT & After WBCT & & Before WBCT & After WBCT & \\
\hline TBARS (nmolMDA/gHb) & $0.308 \pm 0.98$ & $0.317 \pm 0.86$ & $>0.05$ & $0.365 \pm 0.75$ & $0.375 \pm 0.83$ & $<0.05$ \\
\hline CuZnSOD (U/g Hb) & $1898.1 \pm 165.7$ & $2003.3 \pm 198.7$ & $<0.001$ & $2102.18 \pm 213.1$ & $2244.8 \pm 2546$ & $<0.001$ \\
\hline Uric Acid (mg/dl) & $4.8 \pm 0.85$ & $5.52 \pm 0.78$ & $<0.0001$ & $5,5 \pm 0.9$ & $6.0 \pm 0.65$ & $<0.0001$ \\
\hline TAS (mM) & $0.76 \pm 0.24$ & $0.93 \pm 0.36$ & $<0.001$ & $0.78 \pm 0.34$ & $0.91 \pm 0.35$ & $<0.001$ \\
\hline
\end{tabular}

$p$ value refers to difference before WBCT and after WBCT subjects (females and males)

Table 3 The effect of whole body cryostimulation (WBCT) on the level of uric acid (UA), total antioxidant status (TAS), thiobarbituric acid reactive substances (TBARS) in plasma and superoxide dismutase
(SOD), activity in erythrocytes of WBCT study group before (WBCT$0)$ and after 10 days of cryostimulation (WBCT-10) as well as nonWBCT control subjects before (Cont.-0) and 10 days after (Cont.-10)

\begin{tabular}{|c|c|c|c|c|c|c|}
\hline & \multicolumn{2}{|l|}{ Study subjects $(n=46)$} & \multirow[t]{2}{*}{$p$} & \multicolumn{2}{|l|}{ Controls $(n=48)$} & \multirow[t]{2}{*}{$p$} \\
\hline & WBCT-0 & WBCT-10 & & Cont.-0 & Cont. -10 & \\
\hline $\begin{array}{l}\text { TBARS } \\
\text { (nmolMDA/gHb) }\end{array}$ & $0.344 \pm 0.78(0.03)^{\mathrm{a}}$ & $0.352 \pm 0.82(0.04)^{\mathrm{a}}$ & $>0.05$ & $0.303 \pm 0.52(0.03)^{\mathrm{a}}$ & $0.309 \pm 0.43(0.025)^{\mathrm{a}}$ & $>0.05$ \\
\hline CuZnSOD (U/g Hb) & $2060.7 \pm 171.2(123.46)^{\mathrm{a}}$ & $2202.4 \pm 186.7(134.23)^{\mathrm{a}}$ & $<0.001$ & $1922.12 \pm 235.1(56.63)^{\mathrm{a}}$ & $1991.5 \pm 234.4(76.82)^{\mathrm{a}}$ & $>0.05$ \\
\hline Uric acid (mg/dl) & $4.9 \pm 0.82(0.26)^{\mathrm{a}}$ & $5.59 \pm 0.68(0.22)^{\mathrm{a}}$ & $<0.0001$ & $5.09 \pm 0.9(0.29)^{\mathrm{a}}$ & $5.09 \pm 0.75(0.3)^{\mathrm{a}}$ & $>0.05$ \\
\hline TAS (mM) & $0.78 \pm 0.27(0.06)^{\mathrm{a}}$ & $0.92 \pm 0.32(0.09)^{\mathrm{a}}$ & $<0.001$ & $0.86 \pm 0.32(0.06)^{\mathrm{a}}$ & $0.87 \pm 0.34(0.07)^{\mathrm{a}}$ & $>0.05$ \\
\hline
\end{tabular}

a $S E M$ standard error of the mean

We also showed that there was statistically significant increase of $8 \%$ of the TAS in plasma obtained from WBCT study group compared to non-WBCT controls after 10 days $(p<0.001)$ (Fig. 1; Table 3). There was also statistically significant increase of $19 \%$ of TAS in plasma obtained from WBCT study group after 10 days of treatment compared to the initially taken sample in this group $(p<0.001)$ (Fig. 1; Table 2). It is important that no statistically significant increase of the total antioxidant status in plasma obtained from non-WBCT control group after 10 days when compared to the initially taken sample in this group was observed $(p>0.05)$ (Fig. 1).

Finally, our results showed that there was statistically significant increase of $13 \%$ of the level of TBARS in plasma obtained from WBCT study group compared to non-WBCT controls after 10 days $(p<0.001)$ (Fig. 1; Table 3). What must be emphasized there was no statistically significant changes of the level of TBARS in plasma obtained from WBCT study group after 10 days of treatment compared to the initially taken sample in this group (Table 2). We have observed that the level of TBARS in both groups (male and women) after WBCT was higher (by about $2.7 \%$ ) than before WBCT $(p<0.05, p>0.05$ in men and women, respectively) (Table 2). There was no statistically significant increase of the level of TBARS levels in the non-WBCT control group after 10 days in compare to the initially taken sample in this group $(p>0.05)$ (Fig. 1; Table 3).

\section{Discussion}

The response of the human body to cold occurs through changes in the endocrine, circulatory, nervous-muscular, and immunological systems (Kellogg 2006). The cutaneous circulation is a major effecter of human thermoregulation. There are tenfold more cold receptors in dermis compared to heat receptors. Experimental evidence indicates that the early phase of vasoconstriction due to cooling is mainly dependent on neural regulation and that late phase vasoconstriction relies more on non-neural mechanisms (Hampl et al. 2006).

Shivering is the earliest and most primitive response to increase in heat production. This form of thermogenesis consumes large amounts of energy, which is not effective during severe cold (Silva 2006) such as WBCT. Humans have evolved a more efficient and long-lasting form of nonshivering facultative thermogenesis that uses pure metabolic mechanisms to generate heat. The non-shivering thermogenesis has two categories: obligatory and facultative (Kellogg 2006). The facultative thermogenesis is regulated mainly by catecholamines released from adrenals and the 
sympathetic nervous system (Hampl et al. 2006). Obligatory thermogenesis proceeds continuously in all organs and tissues of the body. The most important endocrine factors modulating obligatory thermogenesis are thyroid hormones which increases metabolic rate and thermogenesis (Silva 2006).

Cryogenic temperatures induced vasoconstriction followed by vasodilation after $4 \mathrm{~min}$ which is connected with increasing blood flow seen as skin hyperemia and return to normal skin temperatures (after about $14 \mathrm{~min}$ ). Vasodilatation appears about $4 \mathrm{~min}$ after WBCT and achieves four times higher value than before cryostimulation and can maintain for some hours (Bauer and Skrzek 1999) increasing blood flow and stimulating the elimination of metabolic products. There is a large variation in the individual responses to cold due to factors such as body size, fitness level, amount of subcutaneous fat and sex (Gordon 2001; Gordon et al. 2003). The lowest local skin temperatures after WBCT were recorded in the forearm, $11^{\circ} \mathrm{C}$ and in the calf $12^{\circ} \mathrm{C}$ (Westerlund et al. 2003). In our study, after cryostimulation skin temperature dropped below $13.6^{\circ} \mathrm{C}$ in the back, upper arm, forearm, thigh, and calf. The effects of cryostimulation on rectal temperature were minimal, probably due to the short duration of cryostimulation. Cooling the skin below $20^{\circ} \mathrm{C}$ causes a marked reduction in the production of acetylcholine and in the rate of conduction along cooling nerves, which varies according to the size of fibres, thus producing asynchrony of impulses (Wozniak et al. 2007).

Females have a reduced cold temperature tolerance compared to men because of their lower aerobic capacity. On the other hand, they have more subcutaneous fat, which serves as a thermal insulator (Gordon 2010).

Some authors observe that cryostimulation leads to an increase in plasma ACTH and cortisol, epinephrine and norepinephrine (Leppäluoto et al. 2008), while others have not reported any changes in stress hormones (Zagrobelny et al. 1993). WBCT resulted in decreased hemoglobin and iron in erythrocytes (Banfi et al. 2009) which probably cause decreased testosterone. Iron activates xanthine oxidase while copper deactivates it. Man accumulates iron with age, which decreases testosterone level in blood (ferritin levels rise above $45 \mathrm{ng} / \mathrm{dl}$ ). WBCT resulted in decreased levels of testosterone and estradiol in football players, although there were no changes in the concentration of luteinizing hormone and dehydroepiandrosterone (DHEAS) (Korzonek-Szlacheta et al. 2007). Smolander et al. (2009) showed that ten sessions of WBCT in healthy females did not lead to disorders related to altered secretions of the growth hormone, prolactin, thyrotropin, or thyroid hormone. Thermoregulation induced by low temperatures is associated with an increase in lipid metabolism. The human body uses energy derived mainly from the conversions of carbohydrates and lipids (Vallerand and
Jacobs 1989). The release of norepinephrine from the terminal endings of sympathetic neurons during non-shivering thermogenesis leads to the mobilization of fatty acids from intracellular stores of triacylglycerides and their oxidation in the mitochondria (Florez-Duquet and McDonald 1998). The mechanisms of action of hypothermic protection are not entirely understood. It seems clear that lower temperature protects tissues deprived of oxygen by slowing the rate of cellular damage that occurs due to the formation of free radicals, chemical metabolites, and tissue edema (Miller et al. 2010a, b, c).

In the course of normal human activity, energy production, detoxification of pollutants, and immunologic defense mechanisms, free radicals are produced. Dietary antioxidants (such as proanthocyanidins found in blueberries and bioflavonoids found in citrus fruits), as well as the human antioxidant enzymes (CuZnSOD) and non-enzymatic (UA) provide critical protection against free radical damage. Oxidative stress results when this delicate pro-oxidant/antioxidant equilibrium is disrupted in favor of the pro-oxidant (free radical) state. The enzymatic antioxidant system includes a variety of antioxidant enzymes such as $\mathrm{CuZn}$ SOD, catalase (CAT), and glutathione peroxidase. Antioxidants, whether synthesized endogenously or exogenously administered, are reducing agents that neutralize the oxidative compounds (ROS) before they can cause damage to different biomolecules (Miller et al. 2010a, b, c). There is a need for developing new therapies such as WBCT which can be used as adjuvant antioxidative therapy. In our present study, we determinated TAS in plasma which was distinctly higher $(p<0.001)$ after ten sessions of cryostimulation compared to non-WBCT group. Our observations of suppression of oxidative stress by WBCT are consistent with other reports (Duqué et al. 2005; Siems et al. 1999; Miller et al. 2010a, b, c). Woźniak et al. (2007) shows that cryostimulation induced an increase in the activity of superoxide dismutase (SOD) by $36 \%(p<0.001)$ and glutathione peroxidase $(\mathrm{GPx})$ by $68 \%(p<0.01)$ in the human erythrocytes.

It has been suspected that an adaptation to cold stimuli and the improvement in the body hardening could be related to an increase in the protection against oxidative stress (Duqué et al. 2005). Simes et al. (1999) reported a higher enzymatic protection (i.e., in the increased activity of red blood cells CAT, glutathione peroxidase, SOD) for those who regularly practice winter swimming activities or after heavy endurance physical exercise in comparison with control. This activation can be viewed as an adaptive defensive mechanism to cope with increased oxidative stress.

Our results indicate for the first time that cryostimulation stimulates the antioxidative response of organism via augmentation of SOD activities $(p<0.001)$ and increase of UA level $(p<0.001)$ compared to non-WBCT subjects. UA is a 
natural antioxidant, accounting for upto $60 \%$ of the free radical scavenging activity in human blood. UA can scavenge superoxide, the hydroxyl radical, and singlet oxygen. UA may assist in the removal of superoxide by preventing against the degradation of SOD, the enzyme that is responsible for clearing superoxide from the cell (Kutzing and Firestein 2008).

In our study, we showed that cryostimulation may reduce oxidative stress via a significant increase not only in the level of TAS but also UA in plasma and activity of SOD in erythrocytes. In the present study, lipid peroxidation in erythrocytes measured as TBARS was not statistically increased after WBCT. Other important antioxidants such as glutathione peroxidase should be examined by further research on cryostimulation.

It seems that WBCT may be used as adjuvant therapy in the treatment of diseases with oxidative stress background, since it improves the antioxidant capacity of organism.

Further studies are required to explain the antioxidative mechanisms in humans and determine the short term or long term effects of cryochamber exposure.

Open Access This article is distributed under the terms of the Creative Commons Attribution Noncommercial License which permits any noncommercial use, distribution, and reproduction in any medium, provided the original author(s) and source are credited.

\section{References}

Banfi G, Melegati G, Barassi A (2009) Beneficial effects of whole body cryotherapy on sport haemolysis. J Hum Sport Exerc 24:189-193

Bauer J, Skrzek A (1999) Physiological basis of cryotherapy. Med Sport 15:3-7

Duqué B, Smolander J, Westerlund T, Oksa J, Nieminen R, Moilanen E, Mikkelsson M (2005) Acute and long-term effects of winter swimming and whole-body cryotherapy on plasma antioxidative capacity in healthy women. Scand J Clin Lab Invest 65:395-402

Florez-Duquet M, McDonald RB (1998) Cold-induced thermoregulation and biological aging. Physiol Rev 78:339-358

Gordon CJ (2001) The therapeutic potential of regulated hypothermia. Emerg Med J 18:81-89

Gordon CJ (2010) Response of the thermoregulatory system to toxic insults. Front Biosci (Elite Ed) 2:293-311

Gordon CJ, Fogarty AL, Greenleaf JE, Taylor NAS, Stocks JM (2003) Direct and indirect methods for determining plasma volume during thermoneutral and cold-water immersion. Eur J Appl Physiol 89:471-474

Gregorowicz H, Zagrobelny Z (2007) Systemic cryotherapy. Indications and contraindications, its course, and physiological and clinical results. In: Podbielska H, Stręk W, Miller G (eds) Acta biomedical engineering. Whole body cryotherapy, Indygo Zahir Media, pp 4-16

Hampl R, Starka L, Janský L (2006) Steroids and thermogenesis. Physiol Res 55:123-131

Kellogg DL (2006) In vivo mechanisms of cutaneous vasodilation and vasoconstriction in humans during thermoregulatory challenges. J Appl Physiol 100:1709-1718

Korzonek-Szlacheta I, Wielkoszyński T, Stanek A (2007) Effect of whole body cryotherapy on the levels of some hormones in professional soccer players. Endokrynol Pol 58:27-32
Kutzing MK, Firestein BL (2008) Altered uric acid levels and diseases states. J Pharmacol Exp Ther 324:1-12

Lange U, Uhlemann C, Muller-Ladner U (2008) Serial whole-body cryotherapy in the criostream for inflammatory rheumatic diseases. A pilot study. Med Klin 103:383-388

Leppäluoto J, Westerlund T, Huttunen P, Oksa J, Smolander J, Dugué B (2008) Effects of long-term whole-body cold exposures on plasma concentrations of ACTH, beta-endorphin, cortisol, catecholamines and cytokines in healthy females. Scand J Clin Lab Invest 68:145-153

Lubkowska A, Szygula Z, Klimek AJ (2009) Do sessions of cryostimulation have influence on white blood cell count, level of IL6 and total oxidative and antioxidative status in healthy men? Eur J Appl Physiol 109:67-72

Lubkowska A, Banfi G, Dołęgowska B, Melzi d'Eril VG, Łuczak J, Barassi A (2010) Changes in lipid profile in response to three different protocols of whole-body cryostimulation treatments. Cryobiology 61:22-26

Miller E, Mrowicka M, Malinowska K, Żołyński K, Kędziora J (2010a) Effects of the whole-body cryotherapy on a total antioxidative status and activities of some antioxidative enzymes in blood of patients with multiple sclerosis. J Med Invest 57:168173

Miller E, Mrowicka M, Malinowska K, Saluk-Juszczak J, Kędziora J (2010b) Effects of whole body cryotherapy on oxidative stress in multiple sclerosis patients. J Therm Biol 35:406-410

Miller E, Mrowicka M, Malinowska K, Mrowicki J, Saluk-Juszczak J, Kędziora J (2010c) Effects of whole-body cryotherapy on a total antioxidative status and activities of antioxidative enzymes in blood of depressive multiple sclerosis patients. World J Biol Psychiatry 12:223-227

Miller E, Mrowicka M, Saluk-Juszczak J, Majsterek I (2011) The level of isoprostanes as a non-invasive marker for in vivo lipid peroxidation in secondary progressive multiple sclerosis. Neurochem Res 36:1012-1016

Misra HP, Fridovich J (1972) The role of superoxide anion in the autoxidation of epinephrine and a simple assay for superoxide dismutase. J Biol Chem 247:3170-3175

Mrowicka M, Gancarek P, Bortnik K, Gałecka E, Miller E, Śmigielski J (2008) Activity of superoxide dismutase (CuZn-SOD) in erythrocytes in patients after hips alloplasty. Pol Merkur Lekarski 24:396-398

Perrone S, Szabo M, Bellieni CV, Longini M, Bango M, Kelen D, Treszl A, Negro S, Tataranno ML (2010) Whole body hypothermia and oxidative stress in babies with hypoxic-ischemic brain injury. Pediatr Neurol 43:236-240

Rymaszewska J, Ramsey D, Chladzinska-Kiejna S (2008) Wholebody cryotherapy as adjunct treatment of depressive and anxiety disorders. Arch Immunol Ther Exp 56:63-68

Siems W, Brenke R, Sommerburg O, Grune T (1999) Improved antioxidative protection in winter swimmers. QJM 99:193-198

Silva JE (2006) Thermogenic mechanisms and their hormonal regulation. Physiol Rev 86:435-464

Smolander J, Leppäluoto J, Westerlund T (2009) Effects of repeated whole-body cold exposures on serum concentrations of growth hormone, thyrotropin, prolactin and thyroid hormones in healthy women. Cryobiology 58:275-278

Vallerand AL, Jacobs I (1989) Rates of energy substrates utilization during human cold exposure. Eur J Appl Physiol Occup Physiol 58:873-878

Varon J, Marik PE, Einov S (2011) Therapeutic hypothermia: a stateof-the-art emergency medicine perspective. Am J Emerg Med (Epub ahead of print)

Westerlund T, Oksa J, Smolander J, Mikkelsson M (2003) Thermal responses during and after whole body cryotherapy $\left(-110^{\circ} \mathrm{C}\right)$. J Therm Biol 28:601-608 
Woźniak A, Woźniak B, Drewa G, Mila-Kieszkowska C (2007) The effect of whole-body cryostimulation on prooxidant-antioxidant balance in blood of elite kayakers after training. Eur J Appl Physiol 101:533-537

Yamauchi T (1989) Whole body cryotherapy is method of extreme cold $-175^{\circ} \mathrm{C}$ treatment initially used for rheumatoid arthritis. Z Phys Med Baln Med Klin 15:311
Zagrobelny Z, Halawa B, Niedzielski C, Wawrowska A (1993) The influence of a single whole body cryostimulation on selected hemodynamic indices and hormone concentrations in the serum of healthy individuals. Polski Tygodnik Lekarski 48:303-305 (in Polish) 